\title{
ESTUDO DO COEFICIENTE DE PARTIÇÃO OCTANOL-ÁGUA DE BIFENILAS POLICLORADAS (PCBs) UTILIZANDO PARÂMETROS TOPOLÓGICOS
}

\author{
Lucicleide Ribeiro da Silva \\ Departamento de Química, Instituto de Ciências Exatas, Universidade Federal de Juiz de Fora, 36036-330 Juiz de Fora - MG \\ Márcia M. C. Ferreira* \\ Instituto de Química, Universidade Estadual de Campinas, CP 6154, 13083-970 Campinas - SP
}

Recebido em 19/3/02; aceito em 9/10/02

\begin{abstract}
A STUDY OF OCTANOL/WATER PARTITION COEFFICIENT OF POLYCHLORINATED BIPHENYLS (PCBs) USING TOPOLOGICAL INDICES. A model based on chemical structure was developed for the accurate prediction of octanol/water partition coefficient $\left(\mathrm{K}_{\mathrm{OW}}\right)$ of polychlorinated biphenyls $(\mathrm{PCBs})$, which are molecules of environmental interest. Partial least squares (PLS) was used to build the regression model. Topological indices were used as molecular descriptors. Variable selection was performed by Hierarchical Cluster Analysis (HCA). In the modeling process, the experimental $\mathrm{K}_{\mathrm{OW}}$ measured for 30 PCBs by thin-layer chromatography - retention time (TLC-RT) has been used. The developed model $\left(\mathrm{Q}^{2}=0,990\right.$ and $\left.\mathrm{r}^{2}=0,994\right)$ was used to estimate the $\log \mathrm{K}_{\mathrm{ow}}$ values for the $179 \mathrm{PCB}$ congeners whose $\mathrm{K}_{\mathrm{Ow}}$ data have not yet been measured by TLC-RT method. The results showed that topological indices can be very useful to predict the $\mathrm{K}_{\mathrm{OW}}$.
\end{abstract}

Keywords: topological indices; chemometrics; QSPR.

\section{INTRODUÇÃO}

Os PCBs possuem fórmula geral $\mathrm{C}_{12} \mathrm{H}_{\mathrm{n}} \mathrm{Cl}_{\mathrm{y}}, \mathrm{com} \mathrm{n}=0, \ldots, 10$ e $\mathrm{y}=10$ n. Desta maneira, existem 209 congêneros de PCBs organizados em subcategorias, os homólogos. Estes, por sua vez, são formados por diferentes congêneros que tenham o mesmo número de átomos de cloro ${ }^{1}$. $\mathrm{O}$ número de identificação de cada congênero foi criado por Ballschmiter e Zell ${ }^{2}$, e mais tarde adotado pela IUPAC. Outra maneira de se referir a cada composto é indicando a posição do átomo de cloro no anel, de acordo com a Figura 1, seguida pelo termo CB (ex. 2,2',3-CB).

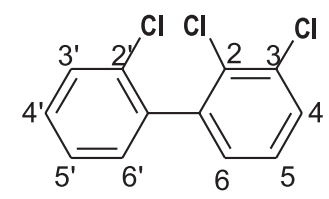

Figura 1. Esquema da molécula 2,2',3-CB

Os PCBs são largamente utilizados na indústria. A versatilidade industrial deles está diretamente relacionada às suas propriedades físicas, tais como resistência à oxidação e redução, excelente propriedade como isolante elétrico, estabilidade térmica e não inflamabilidade $^{3}$. Apesar dessas propriedades físicas importantes, juntamente com seu amplo uso, o descarte dos PCBs é inapropriado, resultando na contaminação do ecossistema global. Nos seres humanos os problemas de saúde que podem ocorrer dependem da dose de exposição ${ }^{4}$. A natureza lipofílica e a persistência dos PCBs contribuem para seu alto potencial de bioacumulação nos níveis mais altos da cadeia alimentar. Isto é, resíduos de PCBs são rotineiramente detectados em peixes e tecido adiposo humano ${ }^{5}$.

Aceita-se cada vez mais que o único método rigoroso para resolver os problemas causados pelos PCBs no meio ambiente consiste em obter as propriedades de todos os congêneros, e tratar cada

*e-mail: marcia@iqm.unicamp.br congênero separadamente. Desta forma, estudar a dinâmica dos PCBs no meio ambiente exige a medida experimental de vários parâmetros. Infelizmente, poucos congêneros são sintetizados em quantidade suficiente para que seja possível a determinação de suas proprieda$\mathrm{des}^{6}$, em função das dificuldades experimentais e do alto custo envolvido. Desta forma, há grandes dificuldades em se obter muitas das propriedades de vários PCBs, e a literatura é escassa em relação a estes dados. Daí a importância e necessidade de busca por modelos teóricos para previsão das mesmas.

O coeficiente de partição octanol-água $\left(\mathrm{K}_{\mathrm{OW}}\right)$ dos PCBs foi a propriedade selecionada para este estudo. $\mathrm{K}_{\mathrm{Ow}}$ é uma medida da lipofilicidade de um composto e é definido como a razão da concentração do mesmo, no equilíbrio, após dissolução em um sistema de duas fases, formadas por dois solventes imiscíveis, água e octanol. A determinação de $\mathrm{K}_{\mathrm{OW}}$ tem sido recomendada pelo programa de evolução de compostos perigosos $(\mathrm{OECD})^{7}$. Nos últimos anos, $\mathrm{K}_{\mathrm{OW}}$ vem sendo muito utilizado em várias áreas, com um número elevado de publicações divulgando a sua correlação com outras propriedades físicas, químicas e biológicas dos compostos, além do sucesso de seu uso na estimativa de fatores de bioconcentração, coeficiente de partição carbono-orgânico, toxicidade e solubilidade ${ }^{8}$. Isto se deve ao fato de $\mathrm{K}_{\mathrm{OW}}$ estar relacionado com a interação do composto em estudo com o meio, no que diz respeito à absorção e transporte. Entre as técnicas experimentais utilizadas para medir $\mathrm{K}_{\mathrm{OW}}$ se destacam: (i) "shake-flask", que é o método clássico de determinação de $\mathrm{K}_{\mathrm{OW}}$; (ii) técnica geradora de coluna (GC); (iii) medidas de cromatografia líquida de fase reversa de alta eficiência e de camada fina (RP-HPLC e RP-TLC). Todas estas técnicas apresentam suas limitações, como a absorção e volatização do soluto $(\mathrm{GC})^{9}$, ou a dificuldade analítica na determinação de $\mathrm{K}_{\mathrm{OW}}$ de substâncias pouco solúveis, pois a determinação da concentração de PCB em água pode ser imprecisa devido à formação de agregados moleculares ${ }^{10}$. Neste trabalho, optou-se por utilizar valores experimentais de $\mathrm{K}_{\mathrm{OW}}$ extraídos da literatura ${ }^{11}$ que fossem medidos pela mesma técnica experimental. Foram encontrados valores experimentais de $\mathrm{K}_{\mathrm{OW}}$ para 30 PCBs obtidos pela medida do tempo de retenção por cromatografia em camada fina (RT-TLC) ${ }^{11}$. 
$\mathrm{K}_{\mathrm{Ow}}$ pode também ser calculado teoricamente utilizando diferentes metodologias: (i) métodos baseados em fragmentos da molécula. Neste caso, $\log \mathrm{K}_{\mathrm{OW}}$ é estimado a partir da contribuição aditiva de grupos funcionais e fragmentos anexados à base molecular ${ }^{12}$; (ii) métodos baseados na contribuição atômica ${ }^{13}$, em que cada átomo tem uma contribuição aditiva para o $\log \mathrm{K}_{\mathrm{OW}}$ da molécula. No caso dos PCBs, estes métodos não produzem resultados precisos, uma vez que os valores obtidos de $\log \mathrm{K}_{\mathrm{ow}}$ para PCBs de um mesmo grupo homólogo têm o mesmo valor; (iii) métodos baseados em modelos que utilizam parâmetros obtidos por meio de cálculos quânticos ${ }^{14}$. Estes métodos apesar de produzirem bons resultados, necessitam de programas e cálculos sofisticados para a otimização da geometria molecular e obtenção dos parâmetros estruturais, eletrônicos, energéticos etc., a serem quantitativamente correlacionados com $\mathrm{K}_{\mathrm{ow}}$. Com base no exposto acima, é visível a importância de se utilizar uma metodologia alternativa para o cálculo de $\mathrm{K}_{\mathrm{OW}}$ que seja eficiente e simples. Neste trabalho, foram utilizados alguns parâmetros topológicos das moléculas de PCBs como variáveis, pois como será mostrado adiante, estes parâmetros são de fácil obtenção, não exigem programas específicos e sofisticados, nem o mesmo tempo computacional que os parâmetros obtidos de cálculos quânticos.

$\mathrm{O}$ estudo das relações quantitativas entre a estrutura química de uma molécula ou uma série de moléculas e sua atividade biológica (QSAR), ou de relações entre a sua estrutura e alguma propriedade físico-química (QSPR), é de grande importância na bioquímica, química medicinal e ambiental. Estes estudos são importantes pois podem fornecer informações sobre as propriedades e características de compostos já existentes, além de subsídios para propor novos compostos com as características de interesse. Esta área teve seu grande avanço na década de 1960, com o trabalho pioneiro de Hansch $^{15}$ e desde então tem sido intensamente aprimorada e utilizada.

Os modelos de estrutura-atividade/propriedade fundamentamse na hipótese de que a estrutura molecular contém as características responsáveis pelas propriedades físicas, químicas e biológicas do composto em questão. Com o avanço tecnológico no que diz respeito a novos computadores e programas, a obtenção destes modelos tornou-se uma alternativa bastante atraente e promissora ${ }^{16}$.

A metodologia geral se inicia com o cálculo de uma série de parâmetros (descritores), onde a grande maioria é obtida a partir da estrutura molecular. O passo posterior ao cálculo dos descritores é a construção de um modelo capaz de relacioná-los com a propriedade/ atividade de interesse. A ferramenta escolhida neste caso é a quimiometria, que dispõe de métodos para a análise de dados multivariados. Os métodos quimiométricos utilizados foram HCA (Hierarchical Cluster Analysis) e PLS (Partial Least Squares) ${ }^{17}$.

Neste trabalho, foi estudada a relação entre estrutura e propriedade de PCBs, aplicando métodos quimiométricos e utilizando como descritores alguns índices topológicos. Os descritores topológicos são obtidos a partir da topologia da molécula e formam um importante conjunto, freqüentemente utilizado em estudos de toxicidade e propriedades biomédicas ${ }^{18}$. Estes parâmetros químicos foram desenvolvidos utilizando a teoria de grafos ${ }^{19}$. Em termos gerais, eles caracterizam a molécula por meio de um único número, e quantificam a estrutura molecular descrevendo características como disposição dos átomos na molécula, forma e ramificação da mesma.

\section{PARTE EXPERIMENTAL}

\section{Parâmetros topológicos}

Para a representação de grafos das moléculas, os átomos são representados como vértices e as ligações como traços ${ }^{20}$. A Figura 2 mostra a representação de grafo do 2-pentanol que contém 5 átomos de carbono, 1 átomo de oxigênio e 6 vértices. A seguir são descritos os índices topológicos que foram utilizados neste trabalho.
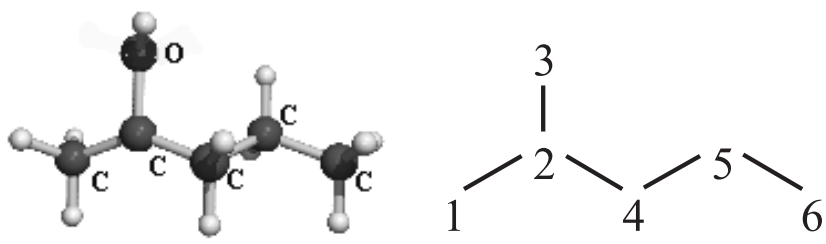

Figura 2. Molécula de 2-pentanol e sua representação de grafo

\section{Índice de Wiener}

O índice de Wiener foi introduzido em $1947^{21}$, sendo o primeiro índice de grafo introduzido na química. Para definir o índice de Wiener, consideremos uma representação de grafo de uma molécula constituída de $k$ átomos onde $v_{1}, v_{2}, \ldots v_{k}$ são os respectivos vértices. A distância entre dois átomos $i$ e $j, D_{i j}$, é dada pela distância entre os vértices $v_{i}$ e $v_{j}$ que é igual ao número de traços (ligações) considerando-se o menor caminho que conecte $v_{i}$ e $v_{j}$. Por exemplo, a menor conexão entre os átomos 1 e 3 da Figura 2 corresponde a dois traços. Portanto $D_{13}=2$. Calculando-se as distâncias entre todos os átomos constituintes da molécula, constrói-se a matriz de distâncias D (6x6) que é dada por:

$\mathbf{D}=\left[\begin{array}{llllll}0 & 1 & 2 & 2 & 3 & 4 \\ 1 & 0 & 1 & 1 & 2 & 3 \\ 2 & 1 & 0 & 2 & 3 & 4 \\ 2 & 1 & 2 & 0 & 1 & 2 \\ 3 & 2 & 3 & 1 & 0 & 1 \\ 4 & 3 & 4 & 2 & 1 & 0\end{array}\right] \begin{aligned} & 12 \\ & 8 \\ & 10 \\ & 14\end{aligned}$

À direita da matriz tem-se o resultado da soma de cada linha, formando uma nova coluna. A soma desta coluna resultante, dividida por dois, é igual ao índice de Wiener, definido na Equação 2 como:

$W=\frac{1}{2} \sum_{i j}\left(D_{i j}\right)$

Para a molécula da Figura 2, o resultado obtido para o índice de Wiener foi $W=32$.

\section{Índice de conectividade}

O conceito de índice de conectividade molecular foi introduzido por Randićc ${ }^{2}$. A idéia é quantificar a estrutura molecular de maneira que fragmentos e subestruturas sejam incorporados como índices numéricos. Primeiro calcula-se, para cada átomo $i$, o descritor de valência $\left(\delta^{v}\right)$ e o descritor de elétrons sigma $\delta$, ambos definidos nas Equações 3 e 4 respectivamente.

$\delta^{\mathrm{v}}=\frac{Z^{\mathrm{v}}-h}{Z-Z^{\mathrm{v}}-1}$

$\delta=\sigma-h$

$\sigma$ é o número de elétrons sigma, $Z^{v}$ é o número de elétrons de valência, $Z$ é o número atômico e $h$ o número de átomos de $\mathrm{H}$ ligados ao átomo $i$. 
Na molécula da Figura 2, de acordo com as Equações 3 e 4, os valores de $\delta$ e $\delta^{\mathrm{v}}$ são:

$\delta_{(-\mathrm{CH} 3)}=\delta_{1}=\delta_{6}=4-3=1 ; \delta_{3(-\mathrm{OH})}=2-1=1$;

$\delta_{(-\mathrm{CH}<)}^{(-\mathrm{CH} 3)}=\delta_{2}=4-1=3 ; \delta_{(-\mathrm{CH} 2-)}=\delta_{4}=\delta_{5}=4-2=2$;

$\delta_{1}^{\mathrm{v}}=\delta_{6}^{\mathrm{v}}=(4-3) /(6-4-1)=1 ; \delta_{3}^{\mathrm{v}}=(6-1) /(8-6-1)=5$;

$\delta_{2}^{\mathrm{v}}=(4-1) /(6-4-1)=3 ; \delta_{4}^{\mathrm{v}}=\delta_{5}^{\mathrm{v}}=(4-2) /(6-4-1)=2$.

Os índices de conectividade molecular são simbolizados por ${ }^{m} \chi_{t}$. As subestruturas de uma molécula e a ordem do índice de conectividade são definidas pela fragmentação da representação de grafo:

a - átomos (ordem zero, $m=0$ );

b - uma ligação (primeira ordem, $m=1$ );

c - fragmentos de duas ligações consecutivas (segunda ordem, $m=$ 2);

d - três ligações contínuas (terceira ordem de caminho, $m=3, t=\mathrm{P}$ );

e - fragmentos incluindo ramificações, três átomos ligados a um átomo central $(m=3, t=\mathrm{C})$;

f - fragmentos incluindo caminho/ramificação, referem-se a quatro ligações adjacentes, com 3 ligações em um átomo central, equivalente à estrutura do isopentano $(m=4-6, t=\mathrm{PC})$;

g - fragmento de cadeia, com anéis de 3, 4, 5, ...átomos $(m=3-6, \ldots$, $t=\mathrm{CH})$.

$\mathrm{O}$ índice de conectividade de valência ${ }^{m} \chi_{t}$ é calculado multiplicando-se os valores de $\delta$ de cada átomo da subestrutura. O inverso da raiz quadrada deste produto é somado com todos os outros produtos de subestruturas de ordem $m$ e tipo $t$ :

${ }^{m} \chi_{t}=\sum\left(\Pi_{k=1}^{m+1} \ddot{a}_{i}\right)^{-0,5}$

O índice de conectividade de valência é obtido usando-se o valor de $\delta^{\mathrm{v}}$ no lugar de $\delta$.

Vejamos agora alguns exemplos do cálculo de ${ }^{m} \chi_{t}$ para a molécula da Figura 2. Para o cálculo de ${ }^{1} \chi$ são considerados os átomos adjacentes existentes na molécula. Os pares são: 1-2, 2-3, 2-4, 4-5 e 5-6. Os valores de $\delta$ utilizados já foram calculados acima. Assim:

${ }^{1} \chi=\left(\delta_{1} \delta_{2}\right)^{-0.5}+\left(\delta_{2} \delta_{3}\right)^{-0,5}+\left(\delta_{2} \delta_{4}\right)^{-0,5}+\left(\delta_{4} \delta_{5}\right)^{-0,5}+\left(\delta_{5} \delta_{6}\right)^{-0,5}=2,770$

Quando se calcula o índice de ordem $2\left({ }^{2} \chi\right)$, deve-se considerar duas ligações consecutivas e, portanto, 3 átomos. Para a molécula em questão, as combinações são: 1-2-3, 1-2-4, 3-2-4, 2-4-5, 4-5-6. Os produtos de $\delta$ dos três átomos são somados, resultando em:

${ }^{2} \chi=\left(\delta_{1} \delta_{2} \delta_{3}\right)^{-0,5}+\left(\delta_{1} \delta_{2} \delta_{4}\right)^{-0,5}+\left(\delta_{2} \delta_{3} \delta_{4}\right)^{-0,5}+\left(\delta_{2} \delta_{4} \delta_{5}\right)^{-0,5}+\left(\delta_{4} \delta_{5} \delta_{6}\right)^{-0,5}=$ 2,183

No cálculo do índice de conectividade de ramificação é necessário identificar no grafo subestruturas semelhantes ao isobutano, em que três átomos estão ligados a um átomo central ( $(Y)$. Neste exemplo existe apenas uma subestrutura, e esta é formada pelos átomos 1-2-3-4:

${ }^{3} \chi_{\mathrm{c}}=\left(\delta_{1} \delta_{2} \delta_{3} \delta_{4}\right)^{-0,5}=0,408$

Para se obter o índice de caminho/ramificação de ordem 4, procura-se na molécula subestruturas que sejam semelhantes à do isopentano ( No caso da molécula deste exemplo, tem-se apenas um fragmento com esta forma, constituído pelos átomos 1-23-4-5. Deve-se notar que este índice procura estruturas semelhantes ao isopentano, mesmo que envolva outros átomos, com exceção do átomo de hidrogênio. Para a molécula considerada, o índice de ordem quatro é:
${ }^{4} \chi_{\mathrm{pc}}=\left(\delta_{1} \delta_{2} \delta_{3} \delta_{4} \delta_{5}\right)^{-0,5}=0,289$

\section{Índice de Harary}

O índice de Harary $\left(H_{D}\right)$ é obtido a partir da matriz de distância D, e recebeu este nome em homenagem a Frank Harary ${ }^{23} . H_{D}$ é definido como:

$H_{D}=\frac{1}{2} \sum_{i j} \frac{1}{D_{i j}}$

Os valores de $D_{i j}$ são os elementos da matriz $\mathbf{D}$ obtidos quando se calculou o índice de Wiener (Equação 1). Então,

$$
\frac{1}{\mathbf{D}}=\left[\begin{array}{lllllll}
0 & 1 & 1 / 2 & 1 / 2 & 1 / 3 & 1 / 4 \\
1 & 0 & 1 & 1 & 1 / 2 & 1 / 3 & 2,583 \\
1 / 2 & 1 & 0 & 1 / 2 & 1 / 3 & 1 / 4 \\
1 / 2 & 1 & 1 / 2 & 0 & 1 & 1 / 2 & 2,583 \\
1 / 3 & 1 / 2 & 1 / 3 & 1 & 0 & 1 & 3,500 \\
1 / 4 & 1 / 3 & 1 / 4 & 1 / 2 & 1 & 0
\end{array}\right] 2,333
$$

Os valores à direita da matriz referem-se à soma de cada linha. $\mathrm{A}$ soma deles dividida por dois, é igual ao índice de Harary e, neste caso, $H_{D}=9$. Este índice é caracterizado por proporcionar uma contribuição menor das ligações terminais do que das ligações internas, ao contrário do índice de Wiener.

\section{Índice eletrotopológico}

Os índices eletrotopológicos são valores numéricos calculados para cada átomo da molécula que contêm informações sobre o ambiente topológico dos átomos e sobre a interação eletrônica devido a outros átomos da molécula ${ }^{24}$.

Para se obter o valor do estado eletrotopológico $\left(E S_{i}\right)$ de um átomo, é necessário primeiro se obter o valor do estado intrínseco $I_{i}$

$$
I_{i}=\frac{\left[\left(\frac{2}{n}\right)^{2} \delta_{i}^{\mathrm{v}}+1\right]}{\delta_{i}}
$$

onde $\delta^{\mathrm{v}}{ }_{i}$ e $\delta_{i}$ foram definidos nas Equações 3 e 4 respectivamente, e $n$ é o número quântico principal.

A perturbação no átomo $i$, originária da presença de todos os outros átomos $j$ da molécula, é uma função da diferença entre os valores intrínsecos e da distância entre $i$ e $j, r_{i j}$, definida como o número de átomos entre $i$ e $j$ (incluindo $i$ e $j$ ). Esta perturbação é definida na Equação 8

$\Delta I_{i}=\sum \frac{I_{i}-I_{j}}{r_{i j}^{2}}$

Finalmente, o estado eletrotopológico do átomo $i$ é dado pela soma dos estados intrínsecos e das perturbações.

$E S_{i}=I_{i}+\Delta I_{i}$

Costuma-se utilizar a soma de $E S_{i}$ da molécula ou de diferentes substituintes como descritor ${ }^{25}$. 
Para a molécula utilizada como exemplo, os valores do estado intrínseco de cada átomo são:

$I_{(-\mathrm{OH})}=I_{3}=\left[(2 / 2)^{2} * 5+1\right] / 1=6 ; I_{(-\mathrm{CH})}=I_{1}=I_{6}=\left[(2 / 2)^{2 * 1+1]} / 1=2 ;\right.$

$I_{(-\mathrm{CH} 2-\mathrm{C}}^{(-\mathrm{OH})}=I_{4}=I_{5}=\left[(2 / 2)^{2 * 2}+1\right] / 2=1,5 ; I_{(-\mathrm{CH}<)}=I_{2}=\left[(2 / 2)^{2 * 3+1] / 3}=1,33\right.$;

$r_{12}=2 ; r_{13}=3 ; r_{14}=3 ; r_{15}=4 ; r_{16}=5 ; r_{23}=2 ; r_{24}=2$;

$r_{25}=3 ; r_{26}=4 ; r_{34}=3 ; r_{35}=4 ; r_{36}=5 ; r_{45}=2 ; r_{46}=3 ; r_{56}=2$

A partir desses valores e dos valores de $r_{i j}$ obtém-se a matriz, cujos elementos são $\left(I_{i}-I_{j}\right) / r_{i j}{ }^{2}$ :

\begin{tabular}{|c|c|c|c|c|c|c|}
\hline 0 & 0,168 & $-0,444$ & 0,056 & 0,031 & 0 & $-0,189$ \\
\hline$-0,168$ & 0 & $-1,168$ & $-0,043$ & $-0,012$ & $-0,027$ & $-1,418$ \\
\hline 0,444 & 1,168 & 0 & 0,500 & 0,281 & 0,160 & 2,553 \\
\hline$-0,056$ & 0,043 & $-0,500$ & 0 & 0 & $-0,056$ & $-0,569$ \\
\hline$-0,031$ & 0,012 & $-0,281$ & 0 & 0 & $-0,125$ & $-0,425$ \\
\hline 0 & 0,027 & $-0,160$ & 0,056 & 0,125 & 0 & 0,048 \\
\hline
\end{tabular}

$\mathrm{Na}$ coluna da direita estão os valores de $\Delta I_{i}$. A partir destes valores são obtidos os índices $E S_{i}$ de acordo com a Equação 9:

$E S_{1}=2-0,189=1,811 ; E S_{2}=1,33-1,418=-0,088 ; E S_{3}=6+$ $2,553=8,553$;

$E S_{4}=1,5-0,569=0,931 ; E S_{5}=1,5-0,425=1,075 ; E S_{6}=2+$ $0,048=2,048$

Portanto a soma dos índices eletrotopológicos é $S s=14,33$.

Os índices topológicos utilizados neste trabalho foram calculados pelo programa Polly ${ }^{26}$ e organizados em uma matriz $\mathbf{X}(209,21)$ (matriz de variáveis independentes). Os símbolos utilizados para identificá-los estão na Tabela 1. Os valores experimentais de $\log \mathrm{K}_{\mathrm{OW}}$ são representados pelo vetor $\mathbf{y}(30,1)$ das variáveis dependentes e estão entre parêntesis na última coluna da Tabela 2 .

O processo geral de modelagem consiste de duas etapas: a primeira é a modelagem propriamente dita, que estabelece uma relação matemática entre $\mathbf{X}$ e y; a segunda é a validação, que otimiza esta relação matemática no sentido de se obter uma melhor descrição da propriedade em estudo. A validação cruzada, excluindo uma amostra de cada vez do conjunto de dados, foi feita para determinar o número de variáveis latentes a ser utilizado no modelo. Concluídas estas etapas, o modelo está apto para prever $\log \mathrm{K}_{\mathrm{ow}}$ daqueles PCBs para os quais não se dispõe de valores experimentais. Antes da modelagem, os dados foram autoescalados, i.e., centrados na média e escalados pela variância ${ }^{17}$. O método de regressão utilizado foi PLS, regressão por mínimos quadrados parciais. A seleção das variáveis para a construção do modelo, foi feita por meio de uma análise hierárquica de agrupamentos $(\mathrm{HCA})^{17}$. Os resultados desta análise são expressos na forma de um dendograma, que agrupa as variáveis de acordo com o grau de similaridade entre elas. Os programas MATLAB $5.1^{27}$ e PLS_Toolbox $2.0^{28}$ foram utilizados para a modelagem. O programa de quimiometria Pirouette versão $3.0^{29}$ foi utilizado para a obtenção do dendograma.

\section{RESULTADOS E DISCUSSÃO}

Inicialmente foram selecionados os descritores que apresentaram uma melhor correlação com $\log \mathrm{K}_{\mathrm{ow}}$. A Figura 3 mostra o dendograma relativo à similaridade entre os índices topológicos $\mathrm{e}$ $\log \mathrm{K}_{\mathrm{Ow}}$. Os ramos horizontais correspondem às variáveis e os números no topo da Figura indicam o índice de similaridade, numa escala que vai de $\mathbf{1}$ (identidade) a $\mathbf{0}$ (nenhuma similaridade). Os descritores que apresentaram maior similaridade com $\log \mathrm{K}_{\mathrm{OW}}$ foram índice de Wiener (W), índices de conectividade e soma dos índices eletrotopológicos (Ss). Excetuando os índices de ramificação de ordem 4 (VC4 e SC4) que formam um conjunto à parte, pode ser observado na Figura 3, uma alta similaridade entre os descritores (variando entre 0,75 e 0,95 ) indicando que existe uma alta correlação

Tabela 1. Índices topológicos utilizados neste trabalho

\begin{tabular}{ll}
\hline $\mathrm{W}$ & $W$ índice de Wiener \\
$\mathrm{HD}$ & $H_{D}$ índice de Harary \\
$\mathrm{Ss}$ & $S S$ soma dos índices eletrotopológicos \\
Sm $(\mathrm{m}=0-6)$ & ${ }^{m} \chi$ índice de conectividade de ordem $m$ \\
$\mathrm{SCm}(\mathrm{m}=3-6)$ & ${ }^{m} \chi_{\mathrm{c}}$ índice de ramificação de ordem $m$ \\
$\mathrm{SPCm}(\mathrm{m}=4-6)$ & ${ }^{m} \chi_{\mathrm{pc}}$ índice de caminho/ramificação de ordem $m$ \\
$\mathrm{Vm}(\mathrm{m}=0-6)$ & ${ }^{m} \chi^{\mathrm{v}}$ índice de conectividade de valência de ordem $m$ \\
$\mathrm{VCm}(\mathrm{m}=3-6)$ & ${ }^{m} \chi_{\mathrm{c}}^{\mathrm{v}}$ índice de ramificação de valência de ordem $m$ \\
\hline
\end{tabular}

Tabela 2. Descritores utilizados na modelagem e os valores previstos e experimentais* de $\log \mathrm{K}_{\mathrm{OW}}$

\begin{tabular}{|c|c|c|c|c|c|c|c|c|c|c|c|c|c|c|c|}
\hline PCB & Estrutura** & $W$ & ${ }^{1} \chi$ & ${ }^{3} \chi_{\mathrm{c}}$ & ${ }^{4} \chi_{\mathrm{pc}}$ & ${ }^{3} \chi_{\mathrm{c}}^{\mathrm{v}}$ & $\log \mathrm{K}_{\mathrm{OW}}$ & PCB & Estrutura** & $W$ & ${ }^{1} \chi$ & ${ }^{3} \chi_{\mathrm{c}}$ & ${ }^{4} \chi_{\mathrm{pc}}$ & ${ }^{3} \chi_{\mathrm{c}}^{\mathrm{v}}$ & $\log \mathrm{K}_{\mathrm{OW}}$ \\
\hline 1 & (2) & 240 & 6,38 & 0,54 & 1,44 & 0,32 & $4,62(\mathbf{4 , 5 6})$ & 54 & $\left(2,2^{\prime}, 6,6^{\prime}\right)$ & 394 & 7,61 & 1,17 & 2,77 & 0,78 & $6,17(\mathbf{5 , 9 4})$ \\
\hline 2 & (3) & 246 & 6,36 & 0,62 & 1,29 & 0,36 & $4,67(4,72)$ & 61 & $(2,3,4,5)$ & 412 & 7,61 & 1,12 & 3,23 & 0,74 & $6,24(\mathbf{6 , 3 9})$ \\
\hline 3 & (4) & 252 & 6,36 & 0,62 & 1,35 & 0,36 & $4,69(\mathbf{4 , 6 9})$ & 70 & $\left(2,3^{\prime}, 4^{\prime}, 5\right)$ & 426 & 7,58 & 1,30 & 2,88 & 0,83 & $6,35(\mathbf{6 , 3 9})$ \\
\hline 4 & $\left(2,2^{\prime}\right)$ & 287 & 6,79 & 0,74 & 1,94 & 0,47 & $5,14(\mathbf{5 , 0 2})$ & 77 & $\left(3,3^{\prime}, 4,4^{\prime}\right)$ & 440 & 7,58 & 1,28 & 3,12 & 0,82 & $6,40(\mathbf{6 , 5 2})$ \\
\hline 5 & $(2,3)$ & 291 & 6,79 & 0,73 & 2,07 & 0,46 & $5,16(5,20)$ & 80 & $\left(3,3^{\prime}, 5,5^{\prime}\right)$ & 426 & 7,54 & 1,49 & 2,19 & 0,92 & $6,37(\mathbf{6 , 5 8})$ \\
\hline 7 & $(2,4)$ & 298 & 6,77 & 0,83 & 1,78 & 0,51 & $5,19(\mathbf{5 , 1 5})$ & 87 & $\left(2,2^{\prime}, 3,4,5^{\prime}\right)$ & 486 & 8,00 & 1,42 & 3,53 & 0,94 & $6,85(\mathbf{6 , 5 8})$ \\
\hline 9 & $(2,5)$ & 293 & 6,77 & 0,83 & 1,79 & 0,51 & $5,18(\mathbf{5 , 1 8})$ & 101 & $\left(2,2^{\prime}, 4,5,5^{\prime}\right)$ & 488 & 7,99 & 1,50 & 3,33 & 0,99 & $6,89(\mathbf{6 , 8 5})$ \\
\hline 11 & $\left(3,3^{\prime}\right)$ & 301 & 6,75 & 0,91 & 1,64 & 0,55 & $5,24(\mathbf{5}, \mathbf{3 4})$ & 116 & $(2,3,4,5,6)$ & 470 & 8,04 & 1,24 & 3,81 & 0,86 & $6,73(\mathbf{6 , 8 5})$ \\
\hline 15 & $\left(4,4^{\prime}\right)$ & 315 & 6,75 & 0,91 & 1,76 & 0,55 & $5,29(\mathbf{5}, \mathbf{2 8})$ & 128 & $\left(2,2^{\prime}, 3,3^{\prime}, 4,4^{\prime}\right)$ & 569 & 8,43 & 1,51 & 4,41 & 1,04 & $7,41(7,44)$ \\
\hline 18 & $\left(2,2^{\prime}, 5\right)$ & 346 & 7,18 & 1,03 & 2,30 & 0,66 & $5,71(\mathbf{5 , 6 4})$ & 153 & $\left(2,2^{\prime}, 4,4^{\prime}, 5,5^{\prime}\right)$ & 573 & 8,40 & 1,69 & 4,00 & 1,13 & $7,49(7,44)$ \\
\hline 28 & $\left(2,4,4^{\prime}\right)$ & 368 & 7,17 & 1,12 & 2,19 & 0,70 & $5,80(\mathbf{5 , 7 4})$ & 185 & $\left(2,2^{\prime}, 3,4,5,5^{\prime}, 6\right)$ & 628 & 8,84 & 1,73 & 4,67 & 1,20 & 7,91(7,93) \\
\hline 29 & $(2,4,5)$ & 354 & 7,18 & 1,01 & 2,47 & 0,65 & $5,74(\mathbf{5}, \mathbf{7 7})$ & 194 & $\left(2,2^{\prime}, 3,3^{\prime}, 4,4^{\prime}, 5,5^{\prime}\right)$ & 738 & 9,25 & 1,90 & 5,53 & 1,32 & $8,58(\mathbf{8 , 6 8})$ \\
\hline 31 & $\left(2,4^{\prime}, 5\right)$ & 362 & 7,17 & 1,12 & 2,20 & 0,7 & $5,79(\mathbf{5}, \mathbf{7 7})$ & 202 & $\left(2,2^{\prime}, 3,3^{\prime}, 5,5^{\prime}, 6,6^{\prime}\right)$ & 702 & 9,25 & 1,94 & 5,19 & 1,35 & $8,48(\mathbf{8}, \mathbf{4 2})$ \\
\hline 37 & $\left(3,4,4^{\prime}\right)$ & 374 & 7,17 & 1,09 & 2,44 & 0,68 & $5,84(\mathbf{5 , 9 0})$ & 206 & $\left(2,2^{\prime}, 3,3^{\prime}, 4,4^{\prime}, 5,5^{\prime}, 6\right)$ & 820 & 9,68 & 2,02 & 6,11 & 1,43 & $9,11(\mathbf{9}, \mathbf{1 4})$ \\
\hline 52 & $\left(2,2^{\prime}, 5,5^{\prime}\right)$ & 412 & 7,58 & 1,32 & 2,65 & 0,85 & $6,30(\mathbf{6}, \mathbf{2 6})$ & 209 & $\left(2,2^{\prime}, 3,3^{\prime}, 4,4^{\prime}, 5,5^{\prime}, 6,6^{\prime}\right)$ & 907 & 10,11 & 2,15 & 6,70 & 1,54 & $9,64(\mathbf{9 , 6 0})$ \\
\hline
\end{tabular}

*Entre parêntesis estão os valores experimentais ${ }^{11}$ de $\log \mathrm{K}_{\mathrm{OW}}$; ** notação conforme Figura 1 


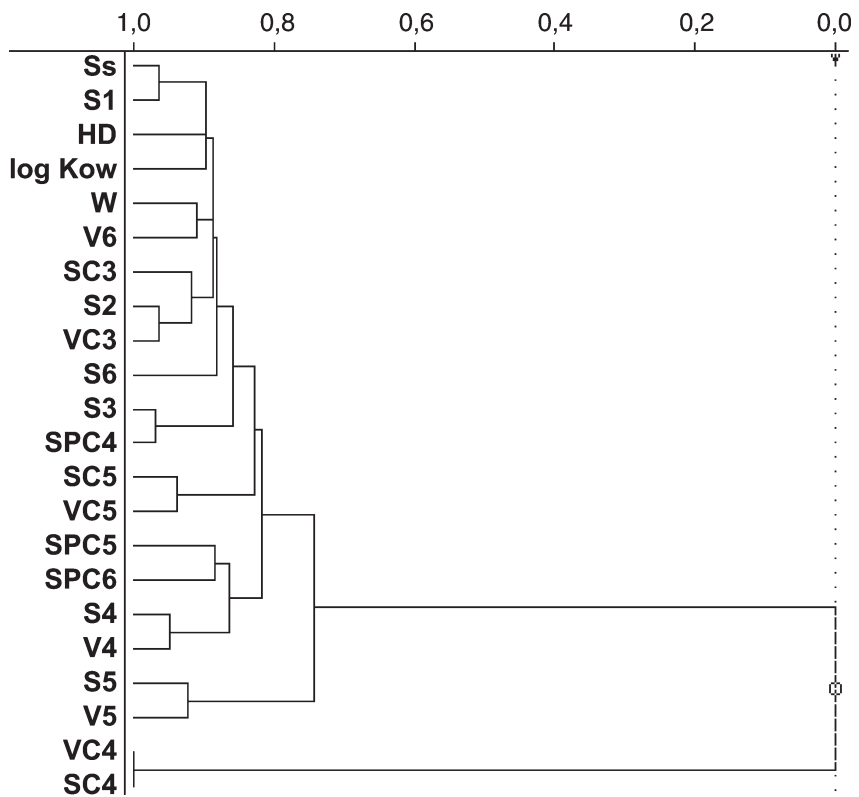

Figura 3. Dendograma obtido da análise de agrupamento hierárquico, mostrando a similaridade entre $\log K_{O W}$ e os índices topológicos

entre os mesmos. Entretanto este fato não se constitui num problema para a construção de modelos de regressão, uma vez que o método utilizado é o de mínimos quadrados parciais (PLS) e não o método tradicional de regressão linear múltipla (MLR) ${ }^{17}$. No método PLS, as variáveis correlacionadas são agrupadas para formar as variáveis latentes contornando assim os problemas de colinearidade, inclusive permitindo a modelagem mesmo quando se tem um reduzido número de compostos (menor que o número de descritores).

A Tabela 2 contém todos os valores dos descritores que foram selecionados, os quais apresentam um comportamento crescente com $\log \mathrm{K}_{\mathrm{ow}}$, ou seja, são diretamente proporcionais (Figura 4). São eles: (i) o índice de Wiener $(W)$. De acordo com Platt, ${ }^{30}$ este índice está relacionado com o volume molecular. Mais precisamente, $W$ representa o quão compacta é a molécula. Quanto mais compactada, menor o valor de $W$ e, portanto, menor o valor de $\log \mathrm{K}_{\mathrm{OW}}$; (ii) o índice de conectividade de primeira ordem $\left({ }^{1} \chi\right)$. Na literatura, o índice de conectividade de primeira ordem é encontrado em diversos modelos, relacionado com o volume molecular ${ }^{31}$, com a área ${ }^{32}$ e com o tempo de retenção de benzenos clorados ${ }^{33}$; (iii) o índice de ramificação de ordem $3\left({ }^{3} \chi_{c}\right)$. Este índice é mais sensível às variações estruturais do que ${ }^{1} \chi$, e descreve principalmente o grau de ramificação da molécula. ${ }^{3} \chi_{\mathrm{c}}$ foi utilizado com sucesso, por exemplo, na estimativa de pressão de vapor de compostos de interesse ambiental ${ }^{34}$; (iv) o índice de quarta ordem de caminho/ramificação $\left({ }^{4} \chi_{\mathrm{pc}}\right)$. Este índice tem sido relacionado à substituição por átomos de cloro em diversos compostos $^{35}$. Também tem sido observado que, em uma mesma clas-
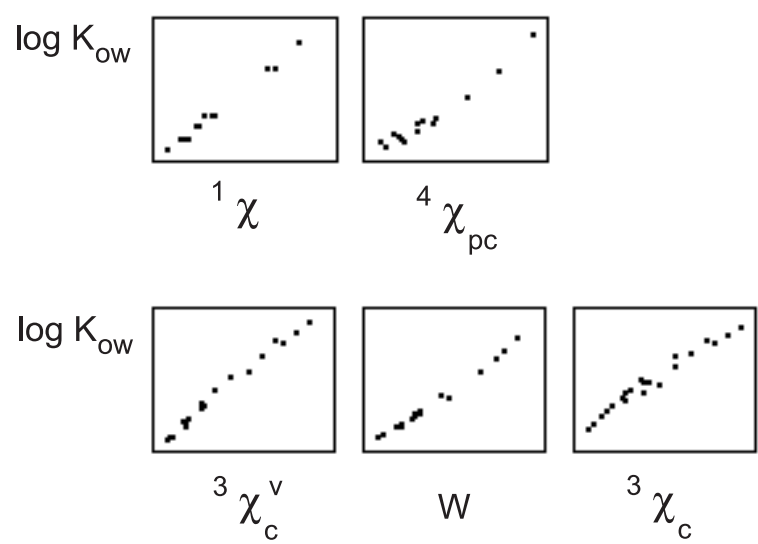

Figura 4. Correlação entre os descritores selecionados e log $K_{\text {OW }}$

se de isômeros, o valor de ${ }^{4} \chi_{\mathrm{pc}}$ aumenta com a proximidade dos átomos de cloro ${ }^{33} ;(v)$ por fim, o índice de ramificação de valência de ordem $3\left({ }^{3} \chi_{\mathrm{c}}^{v}\right)$. O índice ${ }^{3} \chi_{\mathrm{c}}^{\mathrm{v}}$ contém não apenas informações sobre a ramificação da molécula, mas também sobre os elétrons de valência. Então ${ }^{3} \chi_{\mathrm{c}}^{\mathrm{v}}$ pode refletir alguma contribuição eletrônica, decorrente da presença de átomos de cloro. Em virtude da metodologia usada para a construção dos modelos ser PLS, o fato deles serem ou não altamente correlacionados entre si não se constitui numa desvantagem, mas pelo contrário, em vantagem. Como os descritores são utilizados simultaneamente na modelagem, toda a informação relevante intrínseca de cada um é incluída no modelo, enquanto que as informações redundantes são eliminadas.

Foram construídos modelos utilizando três descritores. Os dados estatísticos foram comparados para a escolha do melhor modelo. De acordo com Wold ${ }^{36}$, as condições estatísticas inerentes a um modelo de boa qualidade incluem um desvio padrão residual (s) < 0,30 , o quadrado do coeficiente de correlação $\left(r^{2}\right)>0,90$, e soma dos quadrados residuais (PRESS) $<0,40$. O coeficiente de correlação de validação cruzada $\left(\mathrm{Q}^{2}\right)>0,90$ é indicação de um excelente modelo ${ }^{37}$. Também foram empregados o desvio padrão dos erros de previsão $\left(\mathrm{S}_{\text {PRESS }}\right)$, e o teste de Fischer $(\mathrm{F})$. Neste teste, o valor de F calculado é comparado com o valor tabelado. Uma vez que o valor de F calculado seja maior que o valor de F tabelado, conclui-se que o modelo obtido não é resultado de uma correlação aleatória. Com base nestes critérios, e nos resultados estatísticos para os modelos indicados na Tabela 3, podemos afirmar que todas as combinações apresentadas fornecem modelos de excelente qualidade. Todos eles apresentaram bons coeficientes de regressão com duas variáveis latentes. O modelo escolhido, (4), foi aquele que previu $\mathrm{K}_{\mathrm{ow}}$ para todas as moléculas com o menor resíduo na etapa de validação, e que forneceu o maior valor de F calculado.

No modelo 4, a primeira variável latente (LV) acumula 96,63\% da variância total, e é o resultado da contribuição de todas as variá-

Tabela 3. Dados estatísticos para os modelos de $\log \mathrm{K}_{\mathrm{OW}}$

\begin{tabular}{cccccccc}
\hline Modelo & Descritor & $\mathrm{r}$ & PRESS & $\mathrm{S}_{\text {PRESS }}$ & $\mathrm{s}$ & $\mathrm{Q}^{2}$ & F-calculado \\
\hline 1 & $W,{ }^{1} \chi,{ }^{4} \chi_{\mathrm{pc}}$ & 0,995 & 0,449 & 0,128 & 0,114 & 0,992 & $3,14 \times 10^{3}$ \\
2 & $W,{ }^{3} \chi_{\mathrm{c}},{ }^{3} \chi_{\mathrm{c}}{ }^{\mathrm{v}}$ & 0,997 & 0,361 & 0,112 & 0,114 & 0,993 & $3,88 \times 10^{3}$ \\
3 & ${ }^{3} \chi_{\mathrm{c}},{ }^{1} \chi,{ }^{3} \chi_{\mathrm{pc}}$ & 0,997 & 0,361 & 0,116 & 0,116 & 0,993 & $3,87 \times 10^{3}$ \\
4 & ${ }^{3} \chi_{\mathrm{c}},{ }^{4} \chi_{\mathrm{pc}},{ }^{3} \chi_{\mathrm{c}}{ }^{\mathrm{v}}$ & 0,997 & 0,244 & 0,095 & 0,100 & 0,995 & $5,75 \times 10^{3}$ \\
\hline
\end{tabular}

$\mathbf{F}_{2,27}=5,49$ 
veis. A segunda, descreve uma variância de 3,36\% dos dados originais e apresenta uma contribuição significativa da variável ${ }^{4} \chi_{\mathrm{pc}}$. A terceira variável latente contribui com apenas $0,01 \%$. O valor de PRESS é menor quando se utilizam três LVs, mas a contribuição da terceira LV é muito pequena (menor que 1,0\% para dados autoescalados). Então o modelo foi construído com duas LVs e os resultados obtidos estão na Tabela 4. Na Equação 11 está a equação final obtida para o cálculo de $\mathrm{K}_{\mathrm{OW}}$ a partir de três descritores topológicos juntamente com os resultados estatísticos obtidos na etapa de validação do modelo, em que $n_{a}$ é o número de amostras utilizadas na modelagem. Na Figura 5 são mostrados os valores previstos para todas as moléculas que participaram da modelagem versus os valores experimentais. Estes valores previstos estão também incluídos na última coluna da Tabela 2.

$\log \mathrm{K}_{\mathrm{ow}}=3,35+0,90\left({ }^{3} \chi_{\mathrm{c}}\right)+0,49\left({ }^{4} \chi_{\mathrm{pc}}\right)+1,25\left({ }^{3} \chi_{\mathrm{c}}^{\mathrm{v}}\right)$

$\mathrm{r}^{2}=0,9944 \quad \mathrm{~s}=0,09 \quad \mathrm{Q}^{2}=0,9943$

PRESS $=0,244 \quad \mathrm{~S}_{\text {PRESS }}=0,090 \quad \mathrm{~F}=5,75 \times 10^{3} \quad \mathrm{~F}_{2,27}=5,49 n_{a}=30$

Tabela 4. Porcentagem da variância acumulada em cada variável latente pelo modelo PLS para $\log \mathrm{K}_{\mathrm{OW}}$

\begin{tabular}{lrrrr}
\hline & \multicolumn{2}{c}{ Bloco X } & \multicolumn{2}{c}{ Bloco Y } \\
\#LV & Esta LV & Total & Esta LV & Total \\
\hline $\mathbf{1}$ & 96,63 & 96,63 & 99,43 & 99,43 \\
$\mathbf{2}$ & 3,36 & 99,99 & 0,10 & 99,53 \\
\hline
\end{tabular}

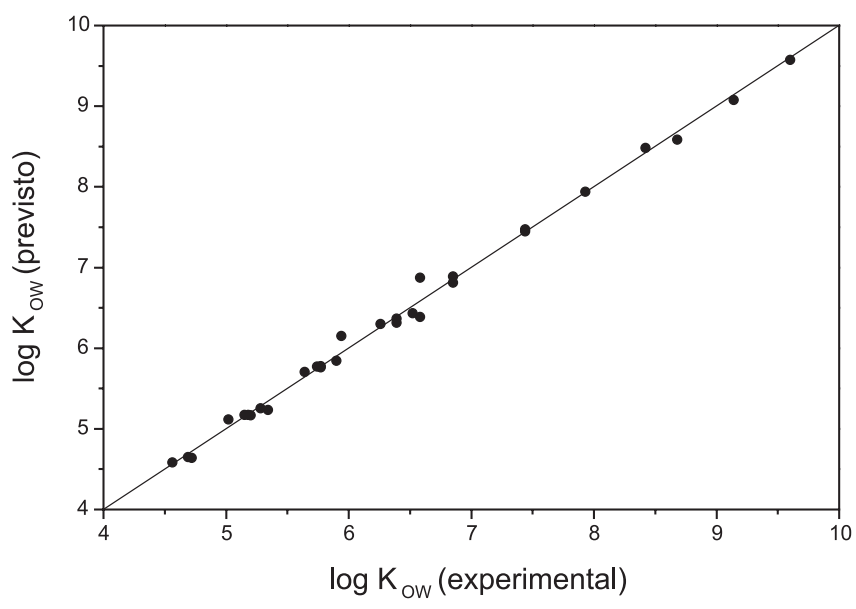

Figura 5. Gráfico dos valores previstos e experimentais de log $K_{\text {OW }}$

Uma maneira de avaliar o desempenho do modelo, seria comparar aos resultados experimentais com os previstos por meio do coeficiente de variabilidade $C V=\frac{\text { desvio padrão dos resíduos }}{\text { média dos valores experimentais }} \times 100$, onde o resíduo é definido como (log $\mathrm{K}_{\text {OWexperimental }}-\log \mathrm{K}_{\text {owprevisto }}$ ). O ideal é que o coeficiente de variabilidade seja uma pequena fração do valor médio experimental. O valor médio dos dados experimentais é 6,37 e o desvio padrão dos resíduos obtido foi de 0,101 , resultando num coeficiente de variabilidade igual a 1,58 (apenas $1,58 \%$ do valor médio dos dados experimentais), que é um valor bastante baixo. Se o desvio padrão dos resíduos for da mesma ordem de magnitude do desvio padrão dos dados experimentais, indica que a Equação 11 não prevê bem os valores experimentais. Idealmente, o valor da razão $\frac{\text { desvio padrão dos valores experimentais }}{\text { desvio padrão dos resíduos }}$ deve ser igual ou maior que 10,0. No presente trabalho, o desvio padrão dos resultados experimentais é de 1,34 dando uma razão de 13,34, o que nos leva a concluir que o modelo obtido é de excelente qualidade e prevê bem os resultados experimentais.

Uma vez obtida e validada a função matemática que relaciona os descritores com a propriedade em questão, esta relação (modelo) é utilizada para previsão. Os resultados previstos para os outros 179 PCBs podem ser obtidos com os autores.

$\mathrm{Na}$ literatura existem alguns resultados de modelos para prever $\log \mathrm{K}_{\mathrm{OW}}$ de PCBs, os quais podem ser comparados com os resultados obtidos neste trabalho. Hawker e colaboradores ${ }^{38}$ correlacionaram $^{\circ}$ $\log \mathrm{K}_{\mathrm{ow}}$ com o $\log \alpha$ (tempo de retenção relativo), o resultado obtido apresentou $\mathrm{r}^{2}=0,90, \mathrm{~s}=0,33 \mathrm{com} n_{a}=30$. Gramatica e colaboradores $^{39}$ correlacionaram $\log \mathrm{K}_{\mathrm{ow}}$ de PCBs com descritores WHIM relacionados ao tamanho molecular, na modelagem foi obtido $\mathrm{r}^{2}=0,95$ $\operatorname{com} n_{a}=139$. Hansen e colaboradores ${ }^{40}$ correlacionaram $\log \mathrm{K}_{\mathrm{OW}}$ com a área da superfície molecular, obtendo $\mathrm{r}^{2}=0,96 \operatorname{com} n_{a}=53$, sendo que os PCBs foram divididos em dois grupos de acordo com o número de átomos de cloro na posição orto, e foi obtida uma equação para cada grupo. Também podemos verificar que a média residual do modelo $4(0,063)$ é menor que a média residual obtida pelo programa CLOGP $(0,141)^{41}$, o qual é adequado para PCBs com um máximo de cinco átomos de cloro na molécula.

Analisando a Equação 11, observa-se que os coeficientes dos índices topológicos utilizados são todos positivos, como esperado (Figura 4). Como mencionado anteriormente, ${ }^{3} \chi_{\mathrm{c}}$ aumenta com o aumento da ramificação na molécula, e no caso dos PCBs esta ramificação é diretamente relacionada à inclusão de átomo de cloro. Por exemplo, a molécula $18\left(2,2^{\prime}, 5-\mathrm{CB}\right) \operatorname{com} \log \mathrm{K}_{\mathrm{ow}}=5,64$ é mais lipofílica que a molécula $4\left(2,2\right.$ '-CB) que possui $\log \mathrm{K}_{\mathrm{OW}}=5,02$. O índice ${ }^{3} \chi_{\mathrm{c}}^{\mathrm{v}}$ foi introduzido por Hall e Randić ${ }^{42}$ para diferenciar os átomos de carbono dos heteroátomos. $\mathrm{O}$ índice ${ }^{3} \chi_{\mathrm{c}}$ é limitado aos elétrons $\sigma$, enquanto que o índice ${ }^{3} \chi_{\mathrm{c}}^{\mathrm{v}}$ inclue todos os elétrons de valência da molécula. Quando se calcula o delta de valência, são considerados os elétrons que podem fazer as ligações $\pi$ e $\sigma$. Os elétrons $\pi$ são mais expostos e mais interativos que os elétrons $\sigma$, sendo mais significativos em interações intermoleculares não covalentes. $\mathrm{O}$ átomo de cloro possui elétrons no orbital $\mathrm{p}$ que podem fazer ligações tipo $\pi$. Então, ${ }^{3} \chi_{\mathrm{c}}^{\mathrm{v}}$ pode estar relacionado à maior disponibilidade de elétrons que podem fazer ligações $\pi$ devido ao aumento de átomos de cloro na molécula. $O$ índice ${ }^{4} \chi_{\mathrm{pc}}$ contém informação sobre a distância entre os átomos de cloro na molécula, quanto maior a proximidade entre os átomos de cloro, maior o valor de ${ }^{4} \chi_{\mathrm{pc}} \mathrm{e}$, portanto, de $\log \mathrm{K}_{\mathrm{OW}}$, por exemplo $\log \mathrm{K}_{\mathrm{OW}}$ do PCB 5 (2,3-CB) é maior que $\log \mathrm{K}_{\mathrm{OW}}$ do PCB 7 (2,4-CB). Nenhum dos descritores considera a posição do átomo de cloro no anel, apenas a disposição dos mesmos. Mas analisando a Tabela 2 é possível notar a influência da posição em que o átomo de cloro está no anel. Por exemplo, o $\log \mathrm{K}_{\mathrm{OW}}$ do PCB 4 com os dois átomos de cloro na posição orto (2,2'-CB), apresenta menor valor $(5,02)$ que o $\log \mathrm{K}_{\mathrm{Ow}}$ do PCB $11(5,34)$ no qual os dois átomos de cloro estão na posição para (3,3'-CB). log $\mathrm{K}_{\mathrm{OW}}$ do PCB $28(5,74)$ é menor que $\log \mathrm{K}_{\mathrm{ow}}$ do PCB $37(5,90)$, sendo que a única diferença é a mudança do átomo de cloro da posição orto $\left(2,4,4\right.$ ' -CB) para a posição para $\left(3,4,4^{\prime}\right.$ 'CB). Até agora foram comparadas as influências dos átomos de cloro nas posições orto e para, mas é possível analisar a influência da posição meta. log de $\mathrm{K}_{\mathrm{OW}}$ do PCB $15(5,28)$ o qual apresenta os átomos de cloro na posição meta (4,4'-CB), é menor que $\log \mathrm{K}_{\mathrm{OW}}$ do PCB $11(5,34)$, no qual os átomos de cloro estão na posição para (3,3'-CB). A importância do átomo de cloro na posição para fica clara quando se observa que $\log \mathrm{K}_{\mathrm{OW}}$ do $\mathrm{PCB} \mathbf{8 0}(6,58)$, com quatro átomos de cloro nas posições para $\left(3,3^{\prime}, 5,5^{\prime}-\mathrm{CB}\right)$, apresenta o mesmo valor que $\log \mathrm{K}_{\mathrm{OW}}$ do PCB 87 que, apesar de apresentar um átomo de cloro a mais, 
possui apenas dois átomos de cloro na posição para (2,2',3,4,5' CB). De fato, átomos de cloro na posição para parecem contribuir mais para a lipofilicidade dos PCBs do que átomos de cloro na posição orto e meta. Sendo a ordem de importância para o aumento da lipofilicidade a seguinte: para, meta e orto.

\section{CONCLUSÕES}

A aplicação apresentada neste trabalho mostra como é possível obter os valores de $\log \mathrm{K}_{\mathrm{ow}}$, uma importante propriedade dos PCBs, para todos os congêneros, utilizando apenas índices topológicos. De uma maneira geral, todos os índices estão relacionados não apenas ao número de átomos de cloro na molécula, mas também à posição e proximidade destes substituintes. Uma das vantagens em se utilizar estes índices está no fato de não ser necessário o conhecimento prévio da estrutura tridimensional da molécula o que, em alguns casos, é difícil de se obter. Destaca-se também a facilidade com que estes índices podem ser calculados, sua natureza não empírica e a sua capacidade em descrever propriedades estruturais locais (por exemplo ${ }^{1} \chi$ ) ou globais (por exemplo $W$ ), assim como propriedade específica relacionada à variação de um determinado substituinte. De um modo geral, a partir de modelos obtidos por meio de índices topológicos é possível prever, por exemplo, o quanto um composto, ainda não sintetizado, pode ser perigoso para o meio ambiente, ou se uma droga pode ou não ser eficiente.

Os resultados obtidos neste trabalho, mostram que o aumento de átomos de cloro dos PCBs pode ser responsável pelo aumento de ramificações e de elétrons $\pi$ na molécula, e a proximidade entre estes átomos, seria então responsável pelo aumento de $\log \mathrm{K}_{\mathrm{OW}}$.

\section{AGRADECIMENTOS}

Agradecemos ao Prof. Dr. S. Basak e à Dra. D. Mills pela utilização do programa POLLY e ao apoio das agências financiadoras, CNPq (L. R. Cirino) e FAPESP (M. M. C. Ferreira).

\section{REFERÊNCIAS}

1. Rachdawong, P.; Christensen, E. R.; Environ. Sci. Technol. 1997, 31, 2686.

2. Ballschmiter, K.; Zell, M.; Fresenius' J. Anal. Chem. 1980, 302, 20.

3. Hutzinger, O.; Safe, S.; Zitko V.; The Chemistry of PCBs, CRC Press: Boca Raton, Fl, 1974.

4. Giesy, J. P.; Kannan, K.; Crit. Rev. Toxicol. 1998, 28, 511.

5. Mullin, M. D.; Pochini, C. M.; McCrindle, S.; Romkes, M.; Safe, S. H.; Safe, L. M.; Environ. Sci. Technol. 1984, 18, 468.

6. Shiu, W. Y.; Mackay, D.; J. Phys. Chem. Ref. Data 1986, 15, 911.

7. Watari, H.; Tanaka, M.; Suzuki, N.; Anal. Chem. 1982, 54, 702.

8. Dearden, J. C.; Environ. Sci. Technol. 1985, 61, 203; Mallholt, H.; Peters, R. H.; Environ. Sci. Technol. 1988, 22, 1479; Ferreira, M. M. C.; Chemosphere 2001, 44, 125.

9. Rapaport, R. A.; Eisenreich, S. J.; Environ. Sci. Technol. 1984, 18, 163.

10. Miller, M. M.; Ghodbane, S.; Wasik, S. P.; Tewari, Y. B.; Martire, D. E.; J. Chem. Eng. Data 1984, 29, 184.
11. Mackay, D.; Shiu, W. Y.; Ma, K. C.; Illustrated Handbook of PhysicalChemical Properties and Environmental Fate for Organic Chemical, Lewis Publishers: Boca Raton, FL, 1993, vol. 3.

12. Leo, A.; Jow, P. Y. C.; Silipo, C.; Hansch, C.; J. Med. Chem. 1975, 18, 865; Nys, G. G.; Rekker, R. F.; Eur. J. Med. Chem. 1974, 9, 361; Suzuki, T.; Kudo,Y.; J. Comput.-Aided Mol. Des. 1990, 4, 155.

13. Broto, P.; J. Chem. Inf. Comput. Sci. 1989, 29, 163; Broto, P.; Moreau, G.; Vandycke, C.; Eur. J. Med. Chem. 1984, 19, 71; Ghose, A. K.; Crippen, G. M.; J. Comput. Chem. 1986, 4, 565.

14. Klopman, G; Iroff, L. D.; J. Comput.Chem. 1981, 2, 157; Bodor, N.; Huang,M.-J.; J. Comput. Chem. 1991, 12, 1182.

15. Hansch, C.; Fujita, T.; J. Am. Chem. Soc. 1964, 86, 1616

16. Franke, R.; Theoretical Drug Design Methods, Elsevier: New York, 1984; Benigni, R.; Richard, M.; Methods 1998, 14, 264; Schultz, T. W.; Seward, J. R.; Sci. Total Environ. 2000, 249, 73.

17. Ferreira, M. M. C.; J. Braz. Chem. Soc. 2002, 13, 742; Ferreira, M. M. C.; Antunes, A. M.; Melgo, M. S.; Volpe, P. L. O.; Quim. Nova 1999, 22, 724; Beebe, K. R.; Pell, P. J.; Seasholtz, M. B.; Chemometrics: A Pratical Guide, Wiley: New York, 1998.

18. Basak, S. C.; Med. Sci. Res. 1987, 15, 605; Raychaudhury, C.; Ray, S. K.; Ghosh, J. J.; Roy, A. B.; Basak, S. C.; J. Comput. Chem. 1984, 5, 581.

19. Trinajstić, N.; Chemical Graph Theory, CRC: Boca Raton, FL, 1992, cap. 3; Neves, P. J.; Costa, J. B. N.; Ndiyae, P. M.; Carneiro, J. W. M.; Quim. Nova 1998, 21, 1 .

20. Kier, L. B.; Hall, L. H.; Molecular Connectivity in Structure-Activity Analysis, John Wiley \& Sons: New York , 1986.

21. Wiener, H.; J. Am. Chem. Soc. 1947, 69, 2636.

22. Randić, M.; J. Am. Chem. Soc. 1975, 97, 6609.

23. Plavšić, D.; Nikoliæ, S.; Trinajstiæ, N.; Mihalić, Z.; J. Math. Chem. 1993, 12,235 .

24. Kier, L. B.; Hall, L. H.; J. Math. Chem. 1991, 7, 229; Hall, L. H.; Mohney, B. K.; Kier, L. B.; J. Chem. Inf. Comput. Sci. 1991, 31, 76

25. Sullivan, J. J.; Jones, A. D.; Tanji, K. K.; J. Chem. Inf. Comput. Sci. 2000, $40,1113$.

26. Basak, S.; Harriss, D.; Magnuson, V.; POLLY, Natural Resources Research Institute: University of Minnesota, Duluth, 1988.

27. Moler, C.; Little, J.; Kleiman, S.; Bangert, S.; MATLAB ${ }^{\mathrm{TM}}$, The Math Works Inc.: Natick, Ma, 1997.

28. Wise, B. M.; Gallagher, N. B.; PLS Toolbox, Eigenvector Technologies: West Richland, WA, 1995

29. Pirouette, Multivariate Data Analysis for IBM PC Systems; Infometrix Inc.: Woodinville, WA, 2001.

30. Platt, J. R.; J. Phys. Chem. 1952, 56, 328.

31. Feng, L.; Zhang, W.; Wang, L.; Wei, L.; Chemosphere 1996, 32, 2505.

32. Sabljić, A.; Environ. Sci. Technol. 1987, 21, 358.

33. Sabljić, A.; J. Chromatogr. 1985, 319, 1.

34. Sacan, M. T. S.; Balcioglu, I. A.; Chemosphere 1998, 36, 451.

35. Kier, L. B.; J. Pharm. Sci. 1980, 69, 1034; Sabljić, A.; Protiæ, M.; Mol. Pharmacol. 1983, 23, 213; Sabljić, A.; J. Chromatogr. 1984, 314, 1.

36. Wold, S.; Quant. Struct.-Act. Relat. 1991, 10, 191.

37. Boyd, R. J.; Boyd, S. L.; J. Am. Chem. Soc. 1992, 114, 1652

38. Hawker, D. W.; Connell, D. W.; Environ. Sci. Technol. 1988, 22, 382.

39. Gramatica, P.; Navas, N.; Todeschini, R.; Chem. Intelligent Lab. Syst. 1998, $40,53$.

40. Hansen, B. J.; Paya-Perez, A. B.; Rahman, M.; Larsen, B. R.; Chemosphere 1999, 39, 2209.

41. Chessells, M.; Hawker, D. W.; de Bruijn, J.; Hermens, J. L. M.; Chemosphere 1990, 18, 457.

42. Kier, L. B.; Hall, L. H.; J. Pharm. Sci. 1983, 72, 1170; Hall, L. H.; Kier, L. B.; J. Mol. Graph. Mod. 2001, 20, 4. 